\title{
Large Chronic Calcified Subdural Hematoma with Empyema-A Long-Term Complication following Shunt Surgery
}

\author{
Sandeep Bhardwaj ${ }^{1} \quad$ Virendra Deo Sinha ${ }^{1} \quad$ Jitendra Singh Shekhawat ${ }^{1} \quad$ Nand Kishore Gora ${ }^{1}$
}

\author{
${ }^{1}$ Department of Neurosurgery, Sawai Man Singh Hospital, Jaipur, \\ Rajasthan, India
}

J Neurosci Rural Pract 2020;11:673-674

Chronic calcified subdural hematoma (CCSDH) is a rare but unusual entity and represents 0.3 to $2.7 \%$ of chronic subdural hematoma. ${ }^{1,2}$ Chronic calcified SDH was first described in literature in 1884 by Von Rokitansky ${ }^{3}$ as autopsy finding. This complication has been rarely reported in literature with CSDH (chronic subdural hematoma) following ventriculoperitoneal (VP) shunt for hydrocephalus. CCSDH are seen in post traumatic subdural hematomas, postmeningitic effusion and in patients of hydrocephalus with VP shunt done. ${ }^{4}$ They are seen usually in children but are seen in all age groups. ${ }^{4}$ This type of lesion, is also known as armored brain or Matrioska head. ${ }^{5}$ Treatment of chronic calcified hematomas is controversial due to limited reexpansion of brain after surgery thus recommending surgery only when acute or progressive neurological symptoms occur. ${ }^{6}$ A 25 -year-old male presented with pus discharge from left parietal wound for past 10 years. Patient also complained of headache on and off for past 10 years. On examination, patient was conscious, oriented, and with no neurological deficit. Patient was oper-

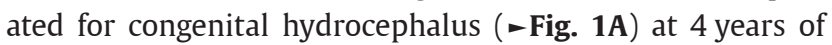
age, for which right sided VP shunt was done in 1996. Patient was doing well until he developed chronic subdural empyema (-Fig. 1B) in 1998, for which patient underwent left parietal and frontal burr hole with evacuation of empyema.

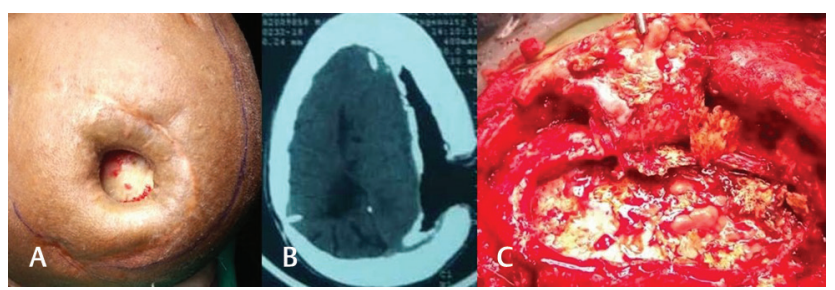

Fig. 1 (A) Noncontrast computed tomography head suggestive of grossly dilated bilateral lateral and third ventricles suggestive of hydrocephalus done in 1996. (B) Noncontrast computed tomography head showing a large left hemispheric hypodense lesion suggestive of subdural empyema with right side VP shunt in situ in 1998. (C) Noncontrast computed tomography brain demonstrating large left hemispheric chronic subdural hematomas with calcification with VP sunt in situ in 2014. VP, ventriculoperitoneal.

\author{
Address for correspondence Virendra Deo Sinha, MBBS, MS, MCh, \\ Department of Neurosurgery, Neurosurgery Department, \\ Sawai Man Singh Hospital Jaipur, 302004, Rajasthan, India \\ (e-mail: sinhavd59@gmail.com).
}

The patient was asymptomatic for almost 10 years. In 2008, patient developed pus discharge from a sinus in left parietal region and underwent left parietal craniotomy with debridement and drainage of empyema cavity. But postoperatively patient continued to have pus discharge from cavity. Patient was lost to follow-up. During that period, he continued antiseptic dressing, till he again reported in 2014. The patient was evaluated with NCCT head, which revealed chronic calcified subdural hematoma, with communicating cavity in left parietal region with VP shunt in situ (-Fig. 1C). Patient was advised surgery but due to unknown reasons he refused it. Patient continued to have pus discharge from left parietal wound. He continued with antiseptic dressing at home along with antibiotics. Patient again reported in 2018 for same complaints and was planned for surgical intervention. NCCT head revealed calcified chronic subdural hematoma with mass effect and midline shift with misplaced tip of VP shunt in situ. Wound culture was sent, antibiotic started according to culture, and when cultures came sterile, patient was taken up for surgery. A large left frontotemporoparietal flap was taken with left frontotemporoparietal craniotomy with removal of chronic calcified subdural hematoma with infected bone flap was done. Calcified chronic subdural hematoma was tightly adherent to inner surface of dura, but arachnoid was intact and not adherent to hematoma. Bone flap was sent for biopsy and culture which came out to be sterile. The postoperative period was uneventful, patient recovered from chronic infection. Postoperatively, patient was kept on empirical course of antibiotics for 6 weeks. Postoperative scan documented reexpansion of brain with resolution of chronic calcified subdural hematoma.

The clinical presentation of chronic calcified subdural hematoma may vary from asymptomatic to headache, vomiting, seizure, growth retardation, paresis, plegia, chronic infection, and transtentorial herniation. ${ }^{7}$ The asymptomatic cases have been due to sufficient cerebrospinal fluid (CSF) drainage that counteracts the raised intracranial pressure. ${ }^{8}$ Chronic calcified subdural hematomas are reported as late 
complications of traumatic brain injury, ${ }^{9}$ postmeningitic subdural effusion, ${ }^{10,11}$ and as a complication of chronic shunting for hydrocephalus. ${ }^{12}$ The underlying mechanism for calcified chronic subdural hematoma is unclear and various underlying metabolic factors are involved in calcification of subdural hematoma in parathyroid disorders have been seen. ${ }^{13}$ Various vascular factors lead to calcification, poor circulation, and absorption in subdural space and vascular thrombosis. ${ }^{14}$ Some local factors that play a role in subdural collections may lead to calcification of hematomas. ${ }^{15}$ The development of calcification in chronic subdural hemorrhage may take months to years. ${ }^{8}$ Subdural collections develop after VP shunt are due to sudden lowering of intracranial pressure and its prevention requires minimal CSF leak at ventricular end insertion, use of programmable/high or medium pressure shunt and close follow up with imaging. ${ }^{16}$ It is advisable to take care of these precautions during VP shunt surgery. Formation of chronic subdural empyema in present case may be due to development of subdural effusion following VP shunt with repeated secondarily infection. ${ }^{17}$ Management of CCSDH is controversial and surgical management is offered to patients with progressive neurological deficit or features of raised intracranial pressure..$^{18}$ Patients who are asymptomatic, elderly, and without any neurological deficit can be managed conservatively. Surgical intervention is required in symptomatic cases as in our case with chronic empyema. Patient should be kept on prolonged antibiotics for 6 weeks to decrease chances of recurrence. However, complete excision has been described but it is difficult to obtain technically. ${ }^{5,19}$ Chronic calcified subdural hematoma with chronic empyema is a rare complication following VP shunt for hydrocephalus. Chronic subdural empyema forms a rare complication following VP shunt which is a cause of delayed morbidity as described in our case. Management of CCSDH is controversial as discussed and should be guided by clinical and neurological presentation. Surgery is beneficial in few subsets of patients with symptoms, rest can be managed conservatively.

\section{Conflict of Interest}

None declared.

\section{References}

1 Yang HZ, Tseng SH, Chen Y, Lin SM, Chen CJ. Calcified chronic subdural haematoma - case report. Tzu Chi Med J 2004;16:261-265

2 Iplikçioğlu AC, Akkaş O, Sungur R. Ossified chronic subdural hematoma: case report. J Trauma 1991;31(2):272-275
3 Von Rokitansky G, Handbuch der Pathologischen Anatomie. Vol. 2. Germany: Wien Braunmuller und Seidel; 1884:717

4 Imaizumi S, Onuma T, Kameyama M, Naganuma H. Organized chronic subdural hematoma requiring craniotomy-five case reports. Neurol Med Chir (Tokyo) 2001;41(1):19-24

5 Sharma RR, Mahapatra A, Pawar SJ, Sousa J, Athale SD. Symptomatic calcified subdural hematomas. Pediatr Neurosurg 1999;31(3):150-154

6 Garg K, Singh PK, Singla R, et al. Armored brain-massive bilateral subdural calcified hematoma in a patient with ventriculoperitoneal shunt. Neurol India 2013;61(5):548-550

7 Dammers R, ter Laak-Poort MP, Maas AIR. Armoured brain: case report of a symptomatic calcified chronic subdural hematoma. J Neurol Neurosurg Psychiatry 2007;78(5):542-543

8 Al Wohaibi M, Russell N, Al Ferayan A. A baby with an armoured brain. CMAJ 2003;169(1):46-47

9 Debois V, Lombaert A. Calcified chronic subdural hematoma. Surg Neurol 1980;14(6):455-458

10 Ludwig B, Nix W, Lanksch W. Computed tomography of the "armored brain" Neuroradiology 1983;25(1):39-43

11 McKay RJ Jr., Morisette RA, Ingraham FD, Matson DD. Collections of subdural fluid complicating meningitis due to haemophilus influenza, type B; a preliminary report. $\mathrm{N}$ Engl J Med 1950;242(1):20

12 Barmeir EP, Stern D, Harel S, Holtzman M, Krije TJ. Calcified subdural haematomas associated with arrested hydrocephalus-late sequelae of shunt operation in infancy. Eur J Radiol 1985;5(3):186-189

13 Boyd DA, Merrell JP. Calcified subdural hematoma. J Nerv Ment Dis 1943;98:609-617

14 Afra D. Ossification of subdural hematoma. Report of two cases. J Neurosurg 1961;18:393-397

15 McLaurin RL, McLaurin KS. Calcified subdural hematomas in childhood. J Neurosurg 1966;24(3):648-655

16 Seyithanoglu H, Guzey FK, Emel E, Ozkan N, Aycan A. Chronic ossified epidural hematoma after ventriculoperitoneal shunt insertion: a case report. Turk Neurosurg 2010;20(4):519-523

17 Kasliwal MK, Sinha S, Kumar R, Sharma BS. Giant hemicranial calcified subdural empyema-unusual complication following ventriculoperitoneal shunt insertion. Indian J Pediatr 2009;76(6):651-652

18 Papanikolaou PG, Paleologos TS, Triantafyllou TM, Chatzidakis EM. Shunt revision 33 years in a patient with bilateral calcified chronic subdural hematomas. Case illustration. J Neurosurg 2008;108:401

19 Moon KS, Lee JK, Kim TS, et al. Contralateral acute subdural hematoma occurring after removal of calcified chronic subdural hematoma. J Clin Neurosci 2007;14(3):283-286 